Freddy Benjamín Naula-Sigua* Diana Jackeline Arévalo-Quishpi*" Jorge Andrés Campoverde-Picón Josselyn Patricia López-González

Recibido: 1 de junio de 2020 Concepto de evaluación: 10 de agosto de 2020

Aprobado: 13 de agosto de 2020

Artículo de investigación (C) 2020 Universidad Católica de Colombia. Facultad de Ciencias Económicas y Administrativas. Todos los derechos reservados

* Máster en Ciencias en Finanzas. Investigador en la Universidad de Cuenca.

Dirección de correspondencia: Facultad de Ciencias Económicas y Administrativas, Universidad de Cuenca, Av. Loja y Av. 12 de Abril (Cuenca, Ecuador). Correo electrónico: freddybenja@gmail.com; fbenjamin.naulas@ucuenca.edu.ec,

(D) https://orcid.org/0000-0003-2218-6216

** Ingeniera comercial. Asesora en Investigación Básica de la Universidad de

Cuenca. Dirección de correspondencia: Facultad de Ciencias Económicas y Administrativas, Universidad de Cuenca, Av. Loja y Av. 12 de Abril (Cuenca, Ecuador). Correo electrónico: diana.arevalo@ucuenca.edu.ec, (D) https://orcid.org/0000-0003-4445-8745

\section{Estrés financiero en el sector manufacturero de Ecuador ${ }^{1}$}

\section{Resumen}

El presente artículo clasifica a las empresas manufactureras del Ecuador en empresas con estrés financiero (ECE) y sin estrés financiero (ESE). Para tal efecto, se clarifica el significado de estrés financiero y el criterio bajo el cual una empresa sería clasificada como ECE o ESE. Además, se recurre a dos modelos ampliamente utilizados en el medio: el análisis discriminante múltiple y la regresión logística, basados en los trabajos previos de Altman y Ohlson, respectivamente. El estudio se enfoca en las empresas del sector manufacturero ecuatoriano durante el periodo 2014-2018. Se destaca que uno de los hallazgos principales es que, en algunos casos, los signos de los coeficientes de los modelos estimados difieren de los modelos originales de Altman y Ohlson. Sin embargo, en ambos casos, las tasas de precisión de este estudio son mayores que las de los modelos originales. Finalmente, se encontró que las microempresas son las que presentan mayor estrés en sentido financiero.

Palabras clave: análisis discriminante múltiple, Ecuador, estrés financiero, manufactura, regresión logística JEL Classification: G33, G30, E00.

\footnotetext{
1 Es artículo fue posible por la gestión del Grupo de Investigación Empresarial y la Dirección de Investigación de la Universidad de Cuenca (Ecuador).
} 
*** Máster en Ciencias en Finanzas. Investigador y analista financiero en Banco Pichincha. Dirección de correspondencia: Banco Pichincha,

Av. 12 de Abril y Av. Solano (Cuenca, Ecuador). Correo electrónico: jorgeandres. campoverde057@comunidadunir.net. (i) https://orcid.org/0000-0002-5386-9688

$\star * \star *$ Ingeniera comercial. Asistente en Investigación Básica de la Universidad de Cuenca. Dirección de correspondencia: Facultad de Ciencias Económicas y Administrativas, Universidad de Cuenca, Av. Loja y Av. 12 de Abril (Cuenca, Ecuador). Correo electrónico: josselyn.lopez@ucuenca.edu.ec. (D https://orcid.org/0000-0003-3795-2593

\section{Financial Distress in the Ecuadorian Manufacturing Sector}

\begin{abstract}
This article classifies Ecuadorian manufacturing companies into companies with and without financial distress. To the effect, the meaning of financial distress (FD) is clarified, as well as the criteria under which a company would be classified as a company with or without FD. Additionally, the study applies two models that are widely used in the middle: multiple discriminant analysis and logistic regression, based on the previous works of Altman and Ohlson, respectively. The research has focused on companies in the Ecuadorian manufacturing sector during the period 2014-2018. As one of the main results, the study found that the signs of the coefficients of the estimated models differ in some cases with respect to those of the original Altman and Ohlson models. Despite this, the precision rates of the present study are higher than those of the original models in both cases. Finally, it was found that microenterprises are the most distressed in a financial sense.

Keywords: Financial distress, multiple discriminant analysis, logistic regression, manufacturing sector, Ecuador.
\end{abstract}




\section{INTRODUCCIÓN}

El sector manufacturero - $-\mathrm{y}$, en específico, su desarrollo- repercute fuertemente en la economía de un país. En los países de Latinoamérica este sector representa, en promedio, el 14,3\% del PIB, convirtiéndose en el cuarto sector con mayor participación (Comisión Económica para América Latina y el Caribe [Cepal], 2017). No obstante, la competitividad, especialmente sudamericana, se ha visto afectada a partir de la década del 2000, debido a que se acentuó su dependencia comercial basada en recursos naturales. Sus capacidades productivas y tecnológicas fueron golpeadas (Abeles, Cimoli y Lavarello, 2017). Esta fragilidad económica fue evidente en la crisis financiera internacional del 2008; la recesión de los países desarrollados golpeó las exportaciones sudamericanas (Cepal, 2008). También la caída del precio del petróleo y de un conjunto de materias primas durante el 2015 impactó de manera negativa a la región. A pesar de que los gobiernos implementaron políticas contracíclicas (Banco Mundial, 2017), la vulnerabilidad económica de la región quedó en evidencia. Lamentablemente, Ecuador no ha sido ajeno a este contexto, aun cuando solo el $8,31 \%$ del total de sus empresas corresponde a la manufactura. Este es uno de los tres sectores que mayores ventas genera y que más aportan al crecimiento económico. En los últimos diez años ha presentado una tasa de $12,69 \%$ de aporte promedio al PIB, desplazando incluso al sector petrolero (Banco Central del Ecuador, 2019).

De lo anterior se deduce que la manufactura, tanto en Latinoamérica como en Ecuador, tiene una importancia crítica para la transición hacia una economía desarrollada (DNIYE, 2018). Sin embargo, el clima en el que se desenvuelve está caracterizado por inestabilidad política, restricciones a los mercados, e ineficiencias burocráticas y en el uso de recursos. Esto, por un lado, limita su potencial y, por otro, agrava el estrés financiero de las empresas de la región (Davig y Hakkio, 2010). En este sentido, un análisis de la probabilidad de estrés financiero (PEF) de las empresas del sector manufacturero resulta pertinente; aportaría directrices para la toma de decisiones, coadyuvando así a la disminución de los costos generados por dicha situación. Con esta finalidad, se emplearon dos modelos altamente populares: Z-Score y O-Score, desarrollados por Altman (1968) y Ohlson (1980), respectivamente. El primer modelo se basa en el análisis discriminante; el segundo utiliza el método de estimación Logit. No obstante, hay que considerar que los coeficientes calculados originalmente fueron obtenidos en una realidad y tiempos distintos a los del contexto nacional. Por lo tanto, es apropiado estimar coeficientes que correspondan con el entorno en el cual se desenvuelven las empresas manufactureras ecuatorianas. 
Freddy Benjamín Naula-Sigua • Diana Jackeline Arévalo-Quishpi • Jorge Andrés Campoverde-Picón • Josselyn Patricia López-González

Así, la muestra fue tomada de la Superintendencia de Compañías del Ecuador, para el periodo 2014-2018.

Se generaron varias especificaciones tanto del modelo de Altman como del de Olson. Una vez estimados, se eligió el modelo que presentó mayor precisión, aplicándolo posteriormente a la muestra total. Se encontró que las provincias de Pichincha y Guayas son las que tienen un mayor número de empresas con estrés (ECE) financiero. Por su parte, considerando el tamaño de las empresas, en el grupo de las microempresas se registró una mayor cantidad de unidades con estrés, casi el doble de las que se presentan en las grandes. Un importante hallazgo se presenta en los signos de los coeficientes de los modelos estimados, los cuales difieren de los modelos originales. Esto, sin duda, se debe al contexto particular ecuatoriano y a su característica de economía en desarrollo. Por lo tanto, la reestimación de los coeficientes, de modo que sean compatibles con el entorno específico del Ecuador, es justificada. En esto radica el principal aporte de este trabajo: coeficientes acordes a una economía no desarrollada.

El resto del contenido del artículo se estructura de la siguiente forma: en la sección 2 se expone la revisión de la literatura; en la sección 3 se explica la metodología; los resultados y la discusión se presentan en la sección 4. Finalmente, se exponen las principales conclusiones.

\section{REVISIÓN DE LA LITERATURA}

\section{Estrés financiero}

El término financial distress, traducido al español, significa 'angustia financiera' o 'aflicción financiera'. No obstante, para efectos del presente artículo y considerando lo que se pretende denotar, se cree más apropiado utilizar el término "estrés financiero" como su equivalente español. Dicho esto, llama la atención que, al realizar la exploración bibliográfica, se encuentren amplias implicaciones para financial distress. Por ejemplo, Fama y French $(1992,1993)$ utilizan la expresión con significados diferentes. En el primero, se relaciona con un alto apalancamiento y las probables dificultades que esto conllevaría. En el segundo artículo se asocia con un elevado ratio book to market. Para Alaka et al. (2018), bancarrota, insolvencia y estrés financieros son indiferentes, todos son sinónimos de falla de la firma. Según Chan y Chen (1991), el estrés financiero significaría directamente la incapacidad de pagar dividendos. Sun et al. (2014) definen el estrés financiero como "la situación en la cual la empresa tiene 
cierto tipo de dificultad financiera". Sin embargo, mencionan que en la literatura clásica estas dificultades pueden englobar la incapacidad para cumplir deudas, obligaciones (Ahmad, 2019; Pozzoli y Paolone, 2017; Shilpa y Amulya, 2017; Beaver, 1966) o dividendos preferenciales, junto con las correspondientes consecuencias, como sobregiros de depósitos bancarios, liquidaciones para el pago de intereses, e incluso entrar en bancarrota. Para Carmichael (1972), las dificultades de cumplir con las obligaciones incluyen insuficiencia de liquidez, insuficiencia de capital y default de la deuda. Para Nandi, Sengupta y Dutta (2019), a largo plazo el estrés financiero implicaría la bancarrota o quiebra. En Herbert (1985), la quiebra es "un procedimiento formal provisto por la ley, que entra en funcionamiento cuando un deudor no puede cumplir con las obligaciones contractuales para hacer ciertos pagos a sus acreedores". Por su parte, Liang et al. (2016) reconocen que estrés financiero, en ciertos mercados, es un término no claro. Aunque el propio autor del Z-Score da a entender que podría implicar falla del negocio, insolvencia, bancarrota o default (Altman y Hotchkiss, 2006), también admite que es difuso en su significado (Altman, 1994; Frydman, Altman y Kao, 1985). De hecho, un ejemplo bastante explícito de su uso variado y de la forma deliberada en la que este se hace se halla en Campbel, Hilscher y Szilagyi (2008).

A pesar de que - como se observó- el término puede ser, en cierta medida, de definición vaga, o puede estar asociado directamente con la bancarrota, el presente trabajo adopta otra perspectiva. Se prefiere la connotación otorgada por el reconocido profesor Stephen Ross, en el sentido de que un elevado apalancamiento financiero podría llevar a dificultades financieras, entre las cuales se vislumbre la bancarrota como una posibilidad, mas no como un hecho dado (Ross, Westerfield y Jordan, 2017). Por lo tanto, a efectos de este trabajo, el estrés financiero es el apalancamiento excesivo, que sobrepasa un umbral que - vale mencionar, por razones que en su momento se darán - es el promedio del ratio de endeudamiento del sector de manufactura. Sin embargo, aunque sí contribuye a alcanzarla, no hay que confundir el estrés financiero con la bancarrota, que es "el estado final en el que las empresas dejan de hacer negocios por dificultades financieras" (Pham Vo Ninh, Do Thanh y Vo Hong, 2018).

Desde el artículo de Altman (1968), en el cual se consideraron empresas manufactureras (EM) de Estados Unidos, para el desarrollo del modelo Z-score, este sector ha sido ampliamente considerado para estudios de estrés financiero. Bae (2012) utiliza al sector manufacturero de Corea del Sur. Chen, Zhang y Zhang (2013), el de China. Pozzoli y Paolone (2017) utilizan las EM italianas. Rifqui y Kanazaki (2016) realizan predicciones de estrés financiero en EM indonesias. Por su parte, Männasoo (2008) encuentra que las EM que están en sus etapas iniciales son más propensas a ser ECE que 
Freddy Benjamín Naula-Sigua • Diana Jackeline Arévalo-Quishpi • Jorge Andrés Campoverde-Picón • Josselyn Patricia López-González

las que están en etapas superiores. Además, las EM aparentemente son más robustas que las empresas de servicios y de comercio. Pindado, Rodrigues y De la Torre (2016) señalan que la elección de deuda de largo plazo está fuertemente condicionada por la búsqueda de una compensación (trade off) entre los beneficios tributarios y los costos ex ante de insolvencia. Asimismo, estos autores apuntan que los préstamos de corto plazo están positivamente afectados por el crecimiento y negativamente asociados con el cash flow. Sayari y Mugan (2017) muestran que el efecto de los distintos ratios financieros utilizados para la predicción del estrés financiero varía entre industrias. De modo que el mismo ratio tiene un signo diferente en distintos grupos de industrias.

Finalmente, en lo que concierne a la literatura, se puede observar que existen varios años de validación, aunque también es usual hallar estudios relacionados con periodos cortos. Por ejemplo, Fernández-Gámez et al. (2020) utilizan solo tres años, aun cuando se trata del modelo Logit Multinivel. Lin (2009) emplea una data correspondiente al periodo 1998-2005; Bae (2012), un periodo levemente inferior. Mselmi, Lahiani y Hamza (2017) utilizan apenas tres años. El estudio llevado a cabo por Laitinen y Laitinen (1998) se aplica tan solo a 82 empresas (41 fallidas y 41 sin fallos), usando datos de 5 años. Por su parte, Ko, Fujita y Li (2017) manejan el periodo 2009-2014 para el modelo de Altman. El caso más interesante quizá sea el de Horváthová y Mokrišová (2020): comparan la eficiencia de DEA y el modelo Logit en la predicción de falla empresariales, solamente con las observaciones de un año. Métodos alternativos, como el de Isik, Jones y Sidorova (2012), también utilizan pocos años (2000 a 2002). Chen et al. (2013) recurre a los datos del periodo 20072010. El estudio realizado por Gregova et al. (2020) contiene información del 2016 al 2018. Como se advierte, es común usar pocos años de validación de la información. En este sentido, para Veganzones y Severin (2020), es más importante la proporción de empresas con fallas con respecto a las sin fallas que el número de años de validación.

\section{Modelos de estrés financiero}

A largo del tiempo se han desarrollado varios modelos de predicción de estrés financiero, que utilizan diferentes herramientas ${ }^{1}$ (Alaka et al., 2018), particularmente susceptibles de análisis a través de ratios financieros (Altman, 2000). Según Beaver (1966), un ratio financiero es "un cociente de dos números, donde ambos [...] consisten en elementos de los estados financieros" (p. 71).

1 Análisis univariante, análisis discriminante múltiple (MDA), logit, probit, árbol de decisiones, redes neuronales artificiales (ANN), máquinas de vectores de soporte (SVM) y razonamiento basado en casos (CBR). 
Sin embargo, a nivel de la literatura, existen cuatro modelos que se pueden considerar relevantes (Pozzoli y Paolone, 2017): a) el Z-score de Altman (1968), basado en análisis discriminante; b) el 0-score de Ohlson (1980), cuya base es una estimación Logit; c) el de Zmijewski (1984), fundado en una estimación probit, y d) el de redes neuronales de Etheridge y Sriram (1997). De ahí en adelante se deriva un conjunto adicional de modelos. En general, los modelos de predicción del estrés se apoyan en medidas obtenidas del mercado o basadas en medidas contables.

Para Beaver, McNichols y Rhie (2005), las medidas obtenidas del mercado presentan ciertas ventajas: a) una riqueza de información basada en los estados financieros de la firma; b) las variables de mercado pueden ser medidas con intervalos de tiempo más pequeños; c) las variables de mercado pueden proveer medidas directas de volatilidad.

No obstante, la información de mercado no siempre está disponible o las empresas no cotizan en bolsa. Consecuentemente, Agarwal y Taffler (2008) exponen dos razones para utilizar medidas contables: a) la bancarrota no es un evento repentino, sino el resultado de varios años de desempeño adverso (esto es capturado por los estados financieros de la firma); b) los préstamos a los cuales las empresas tienen acceso generalmente se basan en información contable.

\section{Modelos univariantes}

Beaver (1966) fue el primero en usar técnicas estadísticas para predecir la quiebra empresarial, a saber: el análisis univariante. Este método genera un punto de corte óptimo para cada uno de los ratios, clasificándolos consecutivamente, y se lo realiza de forma individual (Balcaen y Ooghe, 2006).

\section{Modelos multivariantes}

Altman (1968) observó que el análisis univariante no considera ninguna relación estadística entre las medidas (Bhattacharya, 2007), por lo cual empleó el análisis discriminante múltiple (MDA, por sus siglas en inglés), que resulta útil cuando existe un tipo de relación de dependencia de una variable categórica con respecto a un conjunto de variables independientes métricas (Hair et al., 2014). Así se obtiene una combinación lineal de dos o más variables independientes, que mejor discriminan entre los grupos definidos a priori (Trueck y Rachev, 2009). En su estudio, Altman utilizó 22 ratios, elegidos por su notoriedad en la literatura y su relevancia potencial para el fin buscado. Estos se clasificaron en cinco categorías: liquidez, apalancamiento, 
Freddy Benjamín Naula-Sigua • Diana Jackeline Arévalo-Quishpi • Jorge Andrés Campoverde-Picón • Josselyn Patricia López-González

rentabilidad, solvencia y actividad (Altman, Danovi y Falini, 1988). La función discriminante resultante se denominó Z-Score.

Deakin (1972) comparó a Beaver (1966) y Altman (1968), concluyendo que este modelo es mejor en la predicción de la quiebra un año antes. Sin embargo, el univariante resultó ser mejor en la predicción incluso cinco años antes (Maricica y Georgeta, 2012). Edmister (1972), al aplicar MDA en empresas pequeñas, encontró que un grupo pequeño de ratios tiene mejor nivel de predicción que cualquier ratio tomado de forma individual. En este sentido, Blum (1974) comprobó que los datos contables en bruto generan una predicción más precisa.

Con todo, el modelo de Altman ha gozado de gran popularidad. Ha sido implementado en países como Brasil (Baidya, Ribeiro y Altman, 1979), Argentina (Swanson y Tybout, 1988), Colombia (Valencia Cárdenas et al., 2016), Corea (Eom, Kim y Altman, 1998), Japón (Takahashi, Kurokawa y Watase, 1984), Costa Rica (Hernández Ramírez, 2014) y Francia (Margaine et al., 1974), por mencionar algunos. En todos los casos, se ha adaptado al contexto de cada país. En Ecuador, de igual manera, diversas investigaciones han desarrollado este modelo (Orellana, Reyes y Cevallos, 2018; Pillajo, Salas y Palacios, 2018), aunque no en pleno sentido, pues se tomaron los coeficientes originales, lo cual resulta - cuando menos- inadecuado.

Para Ohlson (1980), el MDA no proporciona la probabilidad de fracaso para una empresa en particular, sino solo una clasificación potencial (Yazdipour, 2011). Además, tiene suposiciones muy restrictivas (Pongsatat, Ramage y Lawrence, 2004). Por ello, este autor utiliza una estimación Logit. La regresión logística permite obtener la probabilidad de que una observación pertenezca a un conjunto determinado, en función del comportamiento de las variables independientes (Mora, 1994). Ohlson desarrolló tres modelos (Dimitras, Zanakis y Zopounidis, 1996); las variables independientes fueron nueve, seleccionadas ad hoc, sin proporcionar ninguna justificación teórica (Wang y Campbell, 2010). En Ecuador, este modelo también se ha utilizado en diversas investigaciones (Espinel, 2016; Osorio, Clavijo y Cevallos, 2018).

\section{Modelos heurísticos}

Entre estos se encuentran las redes neuronales artificiales (ANN), el árbol de decisión, las máquinas de vectores de soporte (SVM) y el razonamiento basado en casos (CBR). Las redes neuronales artificiales (ANN) son "una combinación masivamente paralela de una unidad de procesamiento simple que puede adquirir conocimiento del entorno a través de un proceso de aprendizaje, almacenando el conocimiento en 
sus conexiones" (Guresen y Kayakutlu, 2011). Además, son sensibles al trabajo con datos atípicos (Nyitrai y Virág, 2019). Un método ampliamente utilizado son los árboles de decisión (Müller y Guido, 2016), que divide los datos en subconjuntos con características más homogéneas. Tiene un patrón fácil de entender, rápido y simple, y permite valores atípicos (Wang, Jiang y Wang, 2009).

Por su parte, Kolodner (1993) señala que el CBR es una "metodología basada en el aprendizaje, la memoria y el razonamiento humano, capaz de utilizar la experiencia contextual o casos en la resolución de problemas". Proporciona un modelo computacional que emula relativamente bien el razonamiento humano (Park y Han, 2002). Las SVM son una técnica de Machine Learning (ML), útil en la clasificación y otras actividades de aprendizaje. Según Park y Han (2002), "son modelos de aprendizaje regulados, con aprendizaje relacionado, que investigan la información y perciben patrones". Aunque el método SVM demostró ser superior a métodos similares, como la red neuronal, los enfoques discriminantes lineales múltiples y la regresión logística, no es muy utilizado. Esto se debe a que emplea el principio de minimización del riesgo estructural, por lo que el riesgo empírico de clasificación errónea puede ser alto (Hua et al., 2007).

A pesar de las bondades de los modelos mencionados, ninguna herramienta es predominantemente mejor que el resto (Serrano, Gutiérrez y Bernate, 2019). Es necesario considerar que gran parte de los modelos han surgido en países desarrollados, por lo que al aplicarlos en economías emergentes es necesario tener en cuenta las restricciones que implican estos contextos, por ejemplo, el limitado acceso a la información (Ibarra, 2001).

\section{METODOLOGÍA}

\section{Datos}

Se tomaron los datos de las empresas manufactureras del Ecuador, disponibles en la Superintendencia de Compañías. Para el periodo 2013-2018, se contó inicialmente con los estados financieros de 5527 empresas. ${ }^{2}$ Estos fueron sometidos a un proceso de homogeneización, y se rescató solo a aquellas empresas que cumplieron las siguientes condiciones:

2 Se accedió a la información el 30 de octubre del 2019. 
Freddy Benjamín Naula-Sigua • Diana Jackeline Arévalo-Quishpi • Jorge Andrés Campoverde-Picón • Josselyn Patricia López-González

- Reportaron información completa en el periodo 2013-2018.

- Tienen como estado jurídico activo y están en un proceso de disolución por el numeral 6 del artículo $361^{3}$ de la Ley de Compañías (la razón se detallará más adelante).

- No presentaron irregularidades contables (activo corriente, activo total, pasivo corriente, pasivo total, patrimonio neto igual o menor a cero).

Así, la muestra final comprendió 833 empresas, que ostentan las especificaciones necesarias y suficientes para el desarrollo del estudio.

\section{Variable dependiente}

El presente estudio dicotomiza la situación financiera de las empresas, siendo 1=ECE (empresa con estrés) y $0=E S E$ (empresa sin estrés). Por un lado, como se mencionó, se consideran empresas estresadas financieramente aquellas en proceso de disolución, según el numeral 6 del artículo 361 de la Ley de Compañías. Estas empresas presentan problemas económicos y podrían entrar en liquidación. Por otro lado, puesto que el endeudamiento (pasivo total /activo total) toma en cuenta todas las deudas de todos los vencimientos para todos los acreedores (Ross et al., 2010), se estableció este ratio como criterio adicional para clasificar a las empresas. Cabe señalar que un sobreendeudamiento podría potencialmente acarrear insolvencia y, posteriormente, quiebra (Altman y Hotchkiss, 2006). Así, cualquier empresa que se encuentre sobre el umbral de 0,59058 es clasificada como ECE; las que no sobrepasan este valor son ESE. El valor umbral se obtuvo del promedio del nivel de endeudamiento referencial en el sector manufacturero para el periodo 2014-2018 (Tabla 1).

Utilizar el promedio de un sector o industria como referencia (benhcmark) ha sido una práctica usual en la literatura financiera (Ehrhardt y Brigham, 2007; Foulke, 1968; Palepu, Healy y Bernard, 2003; Ross, Westerfield y Jordan, 2017; Subramanyam y Wild, 2009; Brealey, Myers y Allen, 2011; Koller, Goedhart y Wesseles, 2020). Sin embargo, el comportamiento o explicación que subyace es el de suavizado o alisamiento de los ratios financieros. Al respecto, Gordon (1964) considera que la gerencia de una empresa tiende a reducir/incrementar, por ejemplo, el ratio de crecimiento de ventas, si es que este se encuentra sobre/debajo el promedio de la industria.

\footnotetext{
3 "Las compañías se disuelven por presentar pérdidas del cincuenta por ciento o más del capital social o, cuando se trate de compañías de responsabilidad limitada, anónimas, en comandita por acciones y de economía mixta, por pérdida del total de las reservas y de la mitad o más del capital” (Congreso Nacional, 1999).
} 
Tabla 1.

Endeudamiento y liquidez para 2014-2018

\begin{tabular}{|c|c|c|}
\hline Año & $\begin{array}{c}\text { Ratio endeudamiento de activo } \\
\text { (pasivo total/ activo total) }\end{array}$ & $\begin{array}{c}\text { Liquidez } \\
\text { (activo corriente/pasivo corriente) }\end{array}$ \\
\hline 2014 & 0,6185 & 1,4928 \\
\hline 2015 & 0,5897 & 1,2894 \\
\hline 2016 & 0,5701 & 1,244 \\
\hline 2017 & 0,5947 & 1,5121 \\
\hline 2018 & 0,5799 & 1,2269 \\
\hline
\end{tabular}

Fuente: Superintendencia de Compañías.

Baruch Lev (1969), profesor de la Universidad de Chicago, hace un aporte empírico, generalizando el resto de ratios financieros: encuentra que las empresas utilizan como referencia el promedio de su respectiva industria. En este sentido, considerando el ratio de deuda que determina una ECE y una ESE, la lógica del manejo del promedio sería la siguiente: las empresas deben prestar atención al comportamiento del endeudamiento del resto de empresas a través del promedio de su sector. Si observan que su propio endeudamiento está por debajo del ratio, podrían incrementar su endeudamiento, dado que tienen un margen para hacerlo, con relación al resto de la industria. Por el contrario, si su ratio de endeudamiento resulta ser superior, tenderán a acerarse hacia la media del sector, como medida preventiva. Así evitarán ser catalogadas como potencialmente riesgosas o, en el sentido del presente estudio, como ECE. Resulta entonces válido asumir que el promedio de la industria podría ser considerado como un umbral de la deuda: por encima de este una empresa cualquiera podría ser catalogada como estresada; por debajo, como sin estrés. Se recalca que un perfil que muestre un endeudamiento sobre la media del sector resulta, para efectos de crédito corporativo, digno de atención, cuando menos.

Por otro lado, las empresas resultantes del proceso de depuración -ya señalado en el apartado anterior - sirvieron como inputs tanto para el modelo de Altman como para el de Ohlson. El proceso se muestra en la Figura 1. 
Freddy Benjamín Naula-Sigua • Diana Jackeline Arévalo-Quishpi • Jorge Andrés Campoverde-Picón • Josselyn Patricia López-González

Figura 1.

Proceso de selección de empresas

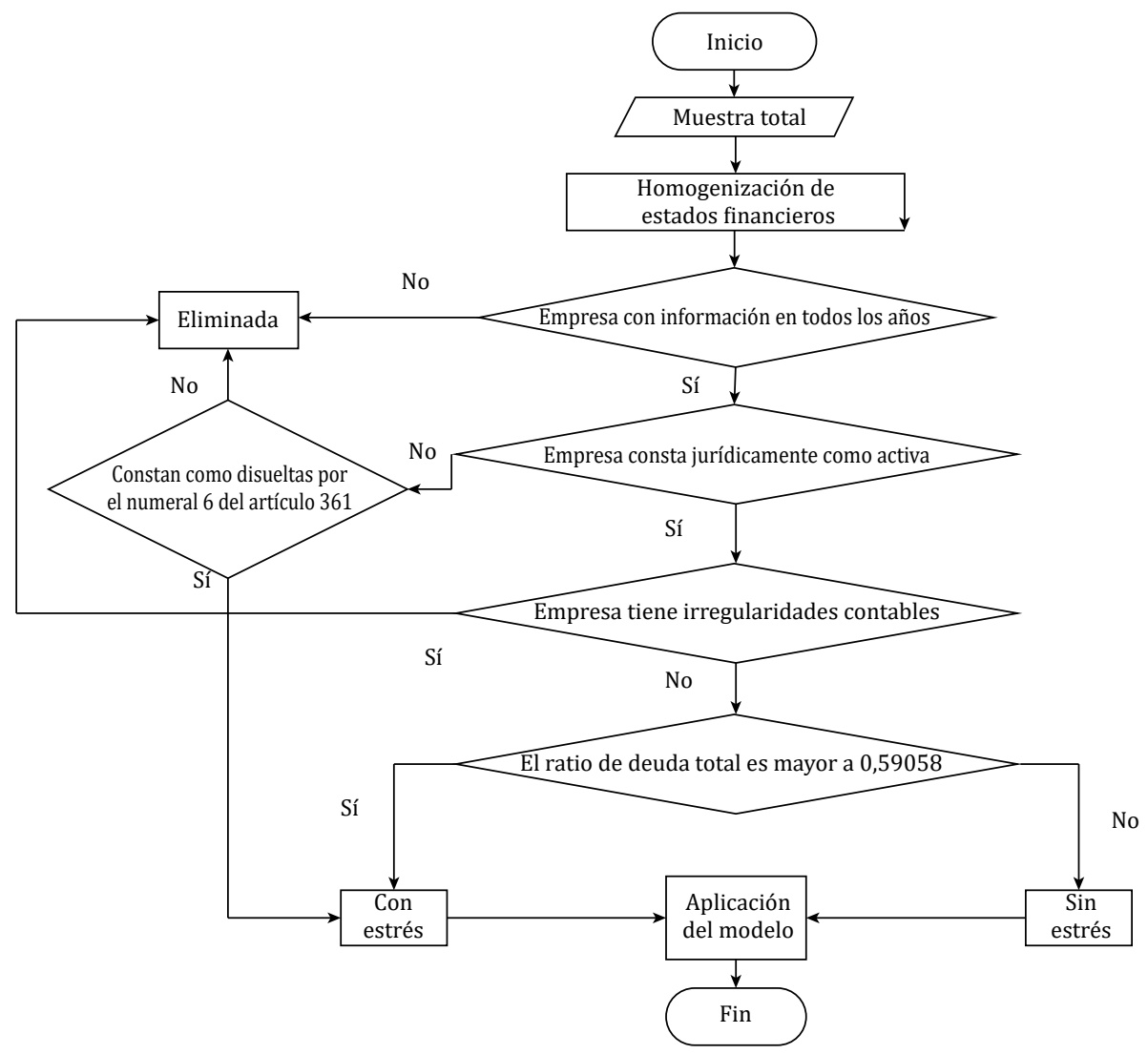

\section{Análisis discriminante múltiple (MDA)}

Como se ha referido, el MDA permite obtener una combinación lineal a partir de dos o más variables independientes (ratios financieros), maximizando la diferencia de los objetos (empresas) en los grupos definidos a priori (ECE y ESE). Se puede trabajar con dos o más grupos (Back et al., 2009). En la ecuación [1] consta la representación lineal de MDA (Altman, 1968):

$$
Z=w_{1} X_{1}+w_{2} X_{2}+\ldots+w_{n} X_{n}
$$


Donde:

$Z=$ Puntaje discrimante

$\mathrm{w}_{1} \ldots \mathrm{w}_{\mathrm{n}}=$ Coeficiente discrimante

$\mathrm{x}_{1} \ldots \mathrm{x}_{\mathrm{n}}=$ Variable discrimante

Las variables discriminantes fueron tomadas de Altman (1993), ya que resultan ser las más adecuadas para el tipo de empresas que se manejan. Estas variables son las siguientes:

$x_{1}($ Liquidez $)=$ Capital de trabajo $/$ Activos totales. Mide los activos corrientes después de haber pagados sus deudas en el corto plazo en relación con los activos totales.

$x_{2}($ Rentabilidad acumulada $)=$ Utilidades retenidas $/$ Activos totales. Cuanto mayor es este índice, mayor es la financiación propia de las empresas. Mide la rentabilidad de los activos totales por cada dólar invertido en estos; esto es, su grado de eficiencia, independientemente de las fuentes de financiación que haya utilizado y de la carga fiscal.

$x_{4}($ Solvencia $)=$ Patrimonio contable $/$ Valor en libros de la deuda. Establece la capacidad de pago de la organización, así como la participación de los acreedores, en términos relativos, dentro de la organización.

$x_{5}($ Actividad $)=$ Ventas $/$ Activos totales. Revela la habilidad de la empresa para generar ventas derivadas del uso de sus activos y, a la vez, su capacidad de gestión para enfrentar las condiciones competitivas del mercado. Para el numerador de este indicador, se utilizó el ingreso por actividades ordinarias de las empresas.

El punto de corte para la segmentación de grupos se calcula de acuerdo con la ecuación [2] (Ramayah et al., 2010):

$\mathrm{Z}_{0}$ : Centroide grupo 0

$$
\text { Punto de corte }=\frac{\mathrm{N}_{1} \mathrm{Z}_{0}+\mathrm{N}_{0} \mathrm{Z}_{1}}{\mathrm{~N}_{1}+\mathrm{N}_{0}}
$$

$\mathrm{N}_{0}$ : Número de observaciones en el grupo 0

$\mathrm{Z}_{1}$ : Centroide grupo 1

$\mathrm{N}_{1}$ : Número de observaciones en el grupo 1 
Freddy Benjamín Naula-Sigua • Diana Jackeline Arévalo-Quishpi • Jorge Andrés Campoverde-Picón • Josselyn Patricia López-González

Para evaluar el poder discriminante que posee una función, se utilizará el valor propio ${ }^{4}$ que evidencia la dispersión de las observaciones. Mientras mayor sea el valor propio de una función, mejor se distinguen los grupos (Chin y Yap, 2012).

\section{Regresión logística (Logit)}

El modelo Logit se expresa en la ecuación [3]:

$$
\mathrm{P}_{\mathrm{i}}=\mathrm{E}\left[\mathrm{Y}_{\mathrm{i}}=1 \mid \mathrm{X}_{\mathrm{i}}\right]=\frac{1}{1+\mathrm{e}^{-\left( \pm+^{2} \mathrm{x}\right)}}=\frac{1}{1+\mathrm{e}^{-\mathrm{Z}}}
$$

Donde $\mathrm{x}$ es el vector de variables independientes de Ohlson, las cuales se exponen a continuación:

$x_{1}($ Tamaño $)=\log ($ Activos totales $/ I P C)$. Implícitamente mide el tamaño de la empresa, a través de la relación existente entre los activos totales y la inflación anual de un país, la cual se calcula a través del índice de precios al consumidor (IPC). ${ }^{5}$

La variable $X_{2}$ utilizada por Ohlson mide la deuda total. Sin embargo, para evitar redundancia con la variable dependiente, se utiliza la deuda de largo plazo: Pasivo no corriente para Patrimonio (Gitman, 1981).

$x_{2}($ Endeudamiento $)=$ Pasivo no corriente $/$ Patrimonio. Indica la relación entre los fondos a largo plazo que suministran los acreedores y los que aportan los dueños de la empresa.

$x_{3}($ Liquidez $)=$ Capital de trabajo $/$ Activos totales . Esta variable fue descrita ya en el modelo de Altman $\left(x_{1}\right)$.

$x_{4}($ Deuda corriente $)=$ Pasivos corrientes $/$ Activos corrientes. Mide la capacidad de la empresa para cumplir con sus obligaciones de corto plazo.

$x_{5}=$ Dummy de solvencia.

$\left\{\begin{array}{l}1 \text { Si pasivo total }>\text { activo total } \\ 0 \text { Si pasivo total } \leq \text { activo total }\end{array}\right.$

$x_{6}($ Rentabilidad $)=$ UAII / Activos totales . Esta variable fue descrita ya en el modelo discriminante $\left(X_{3}\right)$.

\footnotetext{
$4 \quad$ En la literatura anglosajona y alemana es conocida como eigen value.

5 Se utilizó el IPC entregado por el Banco Mundial, teniendo como base el año 2010.
} 
$x_{7}($ Cobertura $)=$ Utilidad operacional $/$ Pasivos totales. Indica la capacidad de cobertura de la deuda por parte de la utilidad operacional.

$\mathrm{x}_{8}=$ Dummy de rentabilidad.

$\left\{\begin{array}{l}1 \text { Si Ingreso Neto } \leq 0 \\ 0 \text { Si Ingreso Neto }>0\end{array}\right.$

$x_{9}($ Ingreso $): \Delta$ Ingresos $_{t}=\left(\operatorname{In}_{t}-\operatorname{In}_{t-1}\right) /\left(\left|\operatorname{In}_{t}\right|+\left|\operatorname{In}_{t-1}\right|\right)$. Variación del ingreso de un periodo a otro.

Tanto en los modelos calculados mediante MDA como con Logit, se calculó la tasa de precisión, es decir, el número de empresas correctamente clasificadas.

\section{RESULTADOS}

\section{Estadística descriptiva}

En la Tabla 2 se presenta la estadística descriptiva de las variables independientes del análisis discriminante y Logit. Con respecto a las variables MDA, en las ESE, el capital de trabajo representa, en promedio, un $32 \%$ del valor de los activos totales; para las ECE, es un 17,8\%. Las primeras tienen un nivel de liquidez mayor que las segundas, lo cual concuerda el estudio de Altman (1968). En el caso de la rentabilidad acumulada $\left(\mathrm{x}_{2}\right)$, las ESE reinvierten, en promedio, un $23,2 \%$ del valor de los activos totales, mientras que las ECE lo hacen en un 13,3\%. En otras palabras, las ESE utilizan mayor financiamiento propio. La rentabilidad $\left(\mathrm{x}_{3}\right)$ es mayor en las ESE que en las ECE. Se trata, concretamente, de 0,1 dólares frente a 0,06 dólares. Este hecho corresponde con los planteamientos de Altman (1968), quien afirma que una empresa en peligro de quiebra ve disminuido el poder de ganancia de sus activos.

En relación con la solvencia $\left(x_{4}\right)$, el patrimonio representa el 177,6\% del pasivo de las ESE; en las ECE, el valor es 35,2\%. Esto significa que las ESE no tienen problemas con sus obligaciones. Respecto a la sctividad $\left(x_{5}\right)$, las ESE rotaron sus activos 1,36 veces, mientras que las ECE lo hicieron 1,53 veces.

En cuanto a las variables correspondientes a Logit, cabe mencionar que $X_{3}$ (liquidez) y $X_{6}$ (rentabilidad) son las mismas que $x_{1}$ (liquidez) y $x_{3}$ (rentabilidad) de MDA, por lo cual se prescinde de su explicación. Ahora bien, con respecto al tamaño $\left(X_{1}\right)$, las ESE presentan, en promedio, un valor de 4,4; las ECE, un valor de 4,1. 
Freddy Benjamín Naula-Sigua • Diana Jackeline Arévalo-Quishpi • Jorge Andrés Campoverde-Picón • Josselyn Patricia López-González

Si consideramos el endeudamiento $\left(X_{2}\right)$, las ESE tienen un ratio de 0,27 y las ECE de 1,94. Por lo tanto, las ECE poseen poca autonomía financiera frente a terceros. En la deuda corriente $\left(X_{4}\right)$, las ESE registran un valor de 0,54; las ECE, 0,82.

De acuerdo con la dummy de solvencia $\left(X_{5}\right)$, todas las ESE fueron solventes. En cuanto a las ECE, el 1,01\% son insolventes, lo que implica que tienen pasivos totales mayores a sus activos totales. A nivel operacional $\left(X_{7}\right)$, las ESE generan una utilidad operacional equivalente al $37,3 \%$ de sus deudas totales; mientras que las ECE, un $12,6 \%$. En cuanto a la rentabilidad $\left(X_{8}\right)$ un 3,91\% de las ECE y un 2,51\% de las ESE no son rentables. Finalmente, de acuerdo con el ingreso $\left(X_{9}\right)$, tanto las ECE como las ESE presentan variaciones entre - 1 y 1 , lo cual obedece a razones internas o externas.

Se puede observar que algunos de los ratios de las ECE, como la liquidez, la rentabilidad acumulada, la solvencia, la rentabilidad y la cobertura de la deuda, son menores a los de las ESE. En el endeudamiento, las ECE presentan un ratio mucho mayor, es decir, poseen mayores obligaciones de largo plazo. Sin embargo, las ECE

Tabla 2.

Estadística descriptiva de las variables MDA y Logit

\begin{tabular}{|c|c|c|c|c|c|c|c|c|c|}
\hline & & \multicolumn{4}{|c|}{ ESE } & \multicolumn{4}{|c|}{ ECE } \\
\hline & & Media & $\Sigma$ & Mínimo & Máximo & Media & $\sigma$ & Mínimo & Máximo \\
\hline \multirow{5}{*}{$\begin{array}{c}\text { Variables } \\
\text { MDA }\end{array}$} & $\mathrm{x} 1$ & 0,32 & 0,219 & $-0,325$ & 0,928 & 0,178 & 0,255 & $-2,828$ & 0,929 \\
\hline & $\mathrm{x} 2$ & 0,232 & 0,17 & 0 & 1,029 & 0,133 & 0,136 & 0 & 2,215 \\
\hline & x3 & 0,1 & 0,09 & 0 & 1,403 & 0,06 & 0,06 & 0 & 0,685 \\
\hline & $\mathrm{x} 4$ & 1,776 & 1,301 & 0,694 & 12,584 & 0,352 & 0,189 & $-0,78$ & 0,693 \\
\hline & $\mathrm{x} 5$ & 1,36 & 0,913 & 0 & 9,223 & 1,539 & 1,191 & 0 & 21,974 \\
\hline \multirow{9}{*}{$\begin{array}{c}\text { Variables } \\
\text { Logit }\end{array}$} & $\mathrm{X} 1$ & 4,403 & 0,794 & 2,162 & 6,759 & 4,095 & 0,754 & 2,162 & 6,436 \\
\hline & $\mathrm{X} 2$ & 0,274 & 0,227 & 0,002 & 1,259 & 1,941 & 4,094 & 0,002 & 39,655 \\
\hline & X3 & 0,32 & 0,219 & $-0,325$ & 0,928 & 0,178 & 0,255 & $-0,325$ & 0,929 \\
\hline & $\mathrm{X} 4$ & 0,541 & 0,449 & 0,022 & 7,304 & 0,821 & 0,615 & 0,022 & 12,329 \\
\hline & $\mathrm{X} 5$ & 0 & 0 & 0 & 0 & 0,01 & 0,1 & 0 & 1 \\
\hline & X6 & 0,1 & 0,09 & 0 & 1,403 & 0,06 & 0,06 & 0 & 0,685 \\
\hline & $\mathrm{X} 7$ & 0,373 & 0,385 & $-3,054$ & 4,388 & 0,126 & 0,154 & $-2,408$ & 1,202 \\
\hline & $\mathrm{X} 8$ & 0,025 & 0,157 & 0 & 1 & 0,039 & 0,194 & 0 & 1 \\
\hline & X9 & $-0,016$ & 0,457 & -1 & 1 & $-0,017$ & 0,541 & -1 & 1 \\
\hline
\end{tabular}

Fuente: elaboración de los autores. 
tienen un ratio de actividad mayor, lo que significaría que generan más ventas. No obstante, el ratio de deuda corriente también es más alto, posiblemente a causa de una mala administración de los activos de corto plazo.

\section{Modelos estimados}

Las Tablas 3 y 4 muestran los modelos estimados a partir MDA y Logit, respectivamente. Se estimaron seis modelos por cada método. El primero de ellos, modelo completo, consideró las 833 empresas durante los cinco años (4165 observaciones). Los cinco modelos restantes toman, respectivamente, solo las observaciones de cada uno de los años.

En la Tabla 3 se muestran los coeficientes discriminantes de los modelos basados en Altman (1993). Entre todos, el de mayor precisión y valor propio es el correspondiente al 2017. Como se advierte, los signos obtenidos en el presente estudio difieren de los del modelo original de Altman.

Antes de la interpretación de los coeficientes, es necesario observar el signo del centroide de cada grupo. Luego, las ESE y las ECE tienden a obtener puntuaciones negativas y positivas, respectivamente, en su función discriminante. Así, en $x_{1}$ (liquidez), $x_{2}$ (rentabilidad acumulada), $x_{3}$ (rentabilidad) y $x_{4}$ (solvencia), el signo es negativo (Lin, 2009). De modo que, en cualquiera de los ratios mencionados, un valor por encima de la media contribuirá a la obtención de un valor negativo en la puntuación discriminante. Esto implica una clasificación sin estrés. En otras palabras, y como es de esperarse, una ESE tiene más liquidez, rentabilidad acumulada, rentabilidad y solvencia. Por su parte, el signo de $x_{5}$ (Actividad) es positivo en todos los modelos (Lin, 2009). Así, un incremento por encima de la media haría más probable una puntuación discriminante positiva, ajustándose más a las características de una ECE. En resumen, las ECE tienen más actividad y tienen menos liquidez, rentabilidad acumulada, rentabilidad y solvencia.

En la primera columna de la Tabla 4 se presentan los coeficientes generados por el estudio original de Ohlson; los obtenidos en este estudio se presentan en el resto de columnas. Como se observa, la precisión de modelo original de Ohlson es inferior a la obtenida por los modelos calculados. Cabe recalcar que $X_{5}$ (Dummy de solvencia) presentó pocas observaciones, por lo que fue omitida. Se advierte que existen ciertos coeficientes con signo distinto al de Ohlson; esto también se presentó en el trabajo de Fernández-Gámez et al. (2020). 
Freddy Benjamín Naula-Sigua • Diana Jackeline Arévalo-Quishpi • Jorge Andrés Campoverde-Picón • Josselyn Patricia López-González

Tabla 3.

Reestimación de los coeficientes del modelo de Altman

\begin{tabular}{|c|c|c|c|c|c|c|c|}
\hline Variable & $\begin{array}{c}\text { Altman } \\
1993\end{array}$ & $\begin{array}{c}\text { Modelo } \\
\text { completo }\end{array}$ & Modelo 2014 & Modelo 2015 & Modelo 2016 & Modelo 2017 & Modelo 2018 \\
\hline x1 & 0,717 & $-0,154$ & $-0,136$ & $-0,152$ & $-0,125$ & $-0,168$ & $-0,185$ \\
\hline x2 & 0,847 & $-0,232$ & $-0,225$ & $-0,175$ & $-0,248$ & $-0,270$ & $-0,232$ \\
\hline x3 & 3,107 & $-0,305$ & $-0,280$ & $-0,261$ & $-0,340$ & $-0,250$ & $-0,390$ \\
\hline x4 & 0,420 & $-0,828$ & $-0,862$ & $-0,856$ & $-0,820$ & $-0,824$ & $-0,776$ \\
\hline x5 & 0,998 & 0,140 & 0,130 & 0,678 & 0,107 & 0,163 & 0,219 \\
\hline $\begin{array}{c}\text { Valor } \\
\text { propio }\end{array}$ & & 0,744 & 0,782 & 0,752 & 0,699 & 0,795 & 0,7489 \\
\hline $\begin{array}{c}\text { Tasa de } \\
\text { precisión }\end{array}$ & $75,55 \%$ & $83,82 \%$ & $84,51 \%$ & $83,31 \%$ & $84,27 \%$ & $85,11 \%$ & $84,15 \%$ \\
\hline N & & 4165 & 833 & 833 & 833 & 833 & 833 \\
\hline n=0 & & 1989 & 350 & 390 & 424 & 416 & 409 \\
\hline n=1 & & 2176 & 483 & 443 & 409 & 417 & 424 \\
\hline $\begin{array}{c}\text { Centroide } \\
\text { Z=0 }\end{array}$ & & $-0,902$ & $-1,038$ & $-0,923$ & $-0,820$ & $-0,891$ & $-0,880$ \\
\hline $\begin{array}{c}\text { Centroide } \\
\text { Z=1 }\end{array}$ & & 0,824 & 0,752 & 0,813 & 0,850 & 0,889 & 0,849 \\
\hline $\begin{array}{c}\text { Punto de } \\
\text { corte }\end{array}$ & & $-0,077$ & $-0,286$ & $-0,110$ & 0,030 & $-0,002$ & $-0,031$ \\
\hline
\end{tabular}

Fuente: elaboración de los autores.

De acuerdo con el criterio BIC y al Pseudo $R^{2}$, el modelo del 2014 es el mejor. Sin embargo, el 2017 es el año que mejor precisión tiene. A continuación se describen y discuten las variables estadísticamente significativas de este modelo. La variable $X_{1}$ (tamaño) muestra un signo negativo, lo cual implica que, si el tamaño de los activos en relación con el índice de precios incrementa, la PEF disminuye. Entonces, mientras más grande es una empresa (medida por sus activos), menor será su probabilidad potencial de quiebra. Por otro lado, $X_{2}$ (endeudamiento) posee un signo positivo; así, a mayor deuda, mayor PEF, lo que es esperable. En lo que corresponde a $X_{3}$ (Liquidez), el signo es negativo, por lo tanto, un incremento en la liquidez disminuye la PEF. La variable $X_{4}$ (deuda corriente) muestra un signo positivo; consecuentemente, su incremento influenciaría positivamente en la PEF. Por su parte, el signo positivo de $X_{6}$ implica que una mayor rentabilidad influye en la PEF. El signo de esta variable difiere del modelo de Ohlson; en el presente estudio, esta variable explica la insolvencia y el estrés financiero, no precisamente la quiebra. 
Considerando que, a decir de Ohlson (1980), una empresa con mayores ingresos tiene mayor capacidad de endeudamiento; que el endeudamiento en niveles excesivos facilitaría la adquisición de activos fijos -y, por ende, contribuiría a una mayor rentabilidad (Molina, 2017) - y que, según Hyz (2019), las empresas necesitan préstamos para continuar generando utilidad en el futuro, se entiende que $X_{6}$ no es malo per se, sino el contexto que podría desatar. Hay que tener en cuenta que el entorno empresarial y sus prácticas, a nivel ecuatoriano, se caracterizan por una aguda falta de planificación y una gestión deficiente. De modo que una rentabilidad excesiva podría, en última instancia, desencadenar un sobreendeudamiento o, para efectos del presente estudio, una tendencia hacia el estrés financiero. Finalmente, $X_{7}$ muestra un signo negativo, lo que significa que una mayor cobertura de la deuda, por parte de la utilidad operacional, disminuye la PEF.

Tabla 4.

Estimación de los coeficientes del modelo de Ohlson

\begin{tabular}{|c|c|c|c|c|c|c|c|c|c|c|c|c|c|}
\hline & $\begin{array}{l}\text { Modelo } \\
\text { Ohlson }\end{array}$ & $\begin{array}{c}\text { Modelo } \\
\text { completo }\end{array}$ & & $\begin{array}{c}\text { Modelo } \\
2014\end{array}$ & & $\begin{array}{c}\text { Modelo } \\
2015\end{array}$ & & $\begin{array}{l}\text { Modelo } \\
2016\end{array}$ & & $\begin{array}{c}\text { Modelo } \\
2017\end{array}$ & & $\begin{array}{c}\text { Modelo } \\
2018\end{array}$ & \\
\hline $\mathrm{X} 1$ & $-0,407$ & $-0,259$ & $* * *$ & $-0,432$ & $* * *$ & $-0,348$ & $* * *$ & $-0,908$ & & $-0,254$ & $*$ & $-0,273$ & $* *$ \\
\hline $\mathrm{X} 2$ & 6,03 & 3,777 & $* * *$ & 3,686 & *** & 3,914 & $* * *$ & 4,200 & $* * *$ & 4,062 & $* * *$ & 3,768 & $* * *$ \\
\hline $\mathrm{X} 3$ & 43 & $-3,546$ & $* * *$ & $-3,412$ & $* * *$ & $-4,738$ & $* * *$ & $-4,110$ & $* * *$ & $-2,680$ & $* * *$ & $-0,492$ & \\
\hline $\mathrm{X} 4$ & 0,0757 & 0,513 & $* *$ & 0,847 & $* *$ & $-0,606$ & & 0,113 & & 1,316 & $* *$ & 2,665 & $* * *$ \\
\hline $\mathrm{X} 6$ & $-2,37$ & 7,381 & $* * *$ & 24,868 & *** & 7,677 & $* * *$ & 1,344 & & 17,272 & $* * *$ & 1,039 & \\
\hline $\mathrm{X} 7$ & 83 & $-4,546$ & *** & $-13,138$ & *** & $-4,115$ & *** & $-3,020$ & $* * *$ & $-8,176$ & $* * *$ & $-1,646$ & *** \\
\hline $\mathrm{X} 8$ & 0,285 & $-1,345$ & $* * *$ & $-0,706$ & & $-1,750$ & $* * *$ & $-0,906$ & & $-1,749$ & $* * *$ & $-1,173$ & * \\
\hline X9 & $-0,521$ & 0,332 & $* * *$ & 0,321 & & 1,068 & $* *$ & 0,284 & & 0,544 & * & $-0,868$ & \\
\hline cons & $-1,32$ & 0,878 & & 1,324 & & & & $-0,436$ & & $-0,901$ & & $-2,208$ & ** \\
\hline BIC & & 3021.256 & & 567,94 & & 8,73 & & 642,18 & & 582,67 & & 660,27 & \\
\hline Pseudo $R_{2}$ & & $48,62 \%$ & & $55,19 \%$ & & $91 \%$ & & $49,17 \%$ & & $54,45 \%$ & & $47,42 \%$ & \\
\hline $\begin{array}{c}\text { Tasa } \\
\text { Precisión }\end{array}$ & $77,86 \%$ & $82,81 \%$ & & $86,06 \%$ & & $83,43 \%$ & & $81,72 \%$ & & $86,34 \%$ & & $84,08 \%$ & \\
\hline $\mathrm{n}$ & & 4141 & & 832,00 & & 833,00 & & 826,00 & & 827,00 & & 323,00 & \\
\hline
\end{tabular}

${ }^{*} \mathrm{p}<0,1 ;{ }^{* *} \mathrm{p}<0,05 ;{ }^{* * *} \mathrm{p}<0,01$

Fuente: elaboración de los autores.

Es importante mencionar que los modelos basados en Olhson obtuvieron una tasa de precisión menor a los que se fundamentaron en Altman. Así, el mejor modelo estimado por MDA obtuvo un $85,11 \%$ de precisión; mientras que su comparable 
Freddy Benjamín Naula-Sigua • Diana Jackeline Arévalo-Quishpi • Jorge Andrés Campoverde-Picón • Josselyn Patricia López-González

Logit, un 86,06\%. Como ya se ha señalado, estos modelos corresponden al 2017 (MDA) y al 2014 (Logit). Tomando los coeficientes de dichos modelos y aplicándolos a la muestra completa, se observó una caída del nivel de precisión: pasó de 85,11\% a $60,62 \%$, para el caso de MDA, y de 86,06\% a 83,07\%, en el caso de Logit. Con base en lo anterior, se utilizó el Modelo 2014 estimado por regresión logística para predecir el estrés financiero de las empresas del sector manufacturero.

\section{Predicción del estrés financiero de las empresas del sector manufacturero del Ecuador}

Los coeficientes del modelo Logit 2014 - que resultó tener el mayor nivel de precisión- fueron aplicados a la muestra completa. Entre los principales resultados, se puede destacar que las regiones con mayores niveles de ECE son Costa y Sierra. En cuanto a provincias, las que muestran más ECE son Pichincha con un $41,25 \%$, Guayas con 40,57\%, Azuay con 4,83\% y Manabí con un 4,34\%. El detalle consta en la Figura 2.

En la Figura 3 se presentan las proporciones correspondientes a ECE, según el tipo de empresa (grande, mediana, pequeña, microempresa) y de acuerdo con el periodo. En el primer caso se observa que, con un 68\%, las microempresas son las que mayor estrés financiero presentan; siguen las empresas pequeñas con un 59\%, las empresas medianas con un $50 \%$, finalmente, las empresas grandes con un $35 \%$. Por otra parte, en el 2015 disminuyó el porcentaje de ECE. Sin embargo, en el 2016 hubo un repunte, con mayor proporción en las microempresas. En el 2017 se reducen, y se mantiene para el 2018. Lo anterior deja ver la fragilidad de las microempresas.

Los resultados expuestos, así como la discusión que sigue a continuación, están, por lo menos, parcialmente en línea con Altman et al. (2017), Chen et al. (2013), Dudley y Ellie (2018), Fernández-Gámez et al. (2020), Ko et al. (2017), Lin (2009), Mselmi et al. (2017) y Pozzoli y Paolone (2017).

\section{Discusión de resultados}

La precisión obtenida en los modelos estimados resultó ser superior a la de los modelos originales de Altman (1968) y Ohlson (1980). Esto se alinea con lo que Oude (2013) afirma respecto a que la precisión de un modelo cambia cuando se aplica en empresas diferentes a las que se utilizaron inicialmente y, por extensión, a diferentes contextos. 
Figura 2.

Empresas manufactureras con estrés financiero por provincia

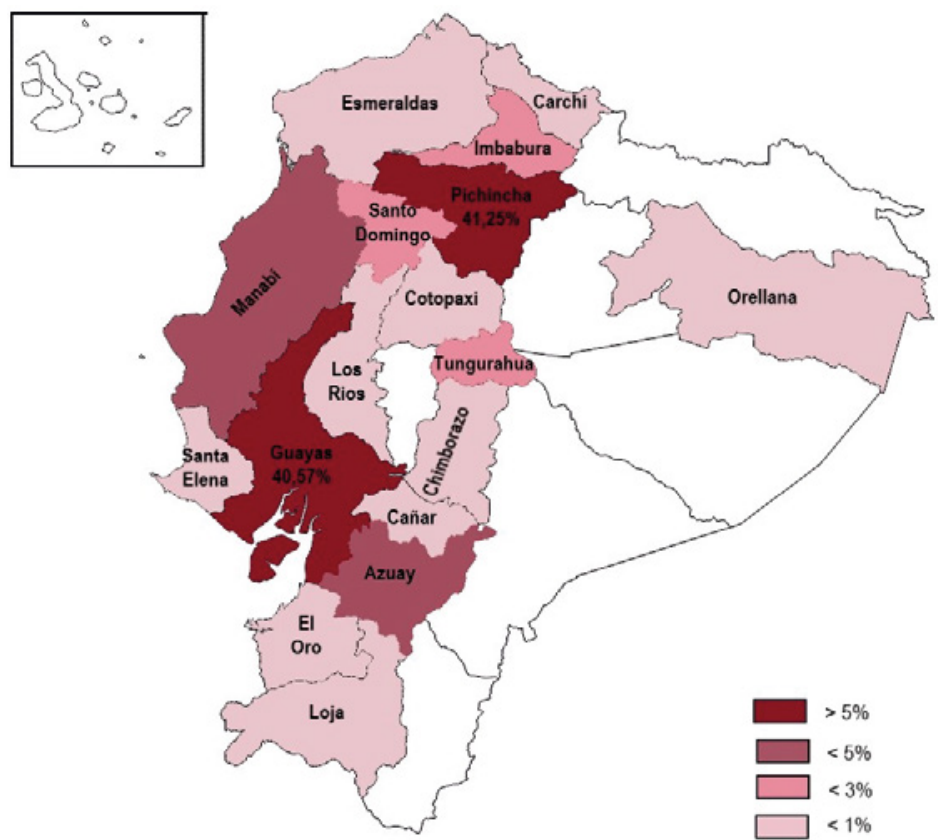

Fuente: elaboración de los autores.

Figura 3.

\section{ECE según tipo de empresas y periodo (muestra completa)}

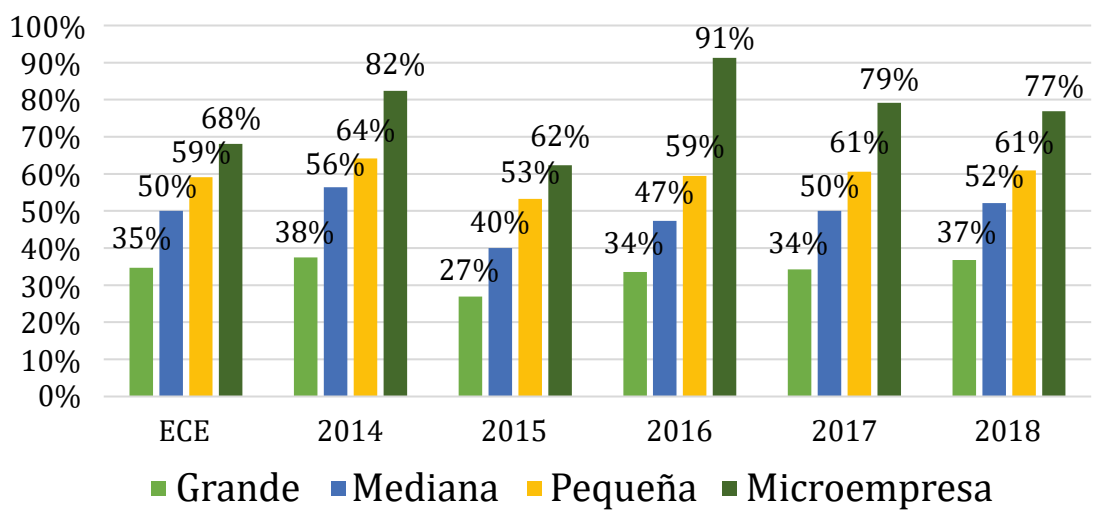

Fuente: elaboración de los autores. 
Freddy Benjamín Naula-Sigua • Diana Jackeline Arévalo-Quishpi • Jorge Andrés Campoverde-Picón • Josselyn Patricia López-González

Los mejores modelos para MDAy Logit son los del 2014 y el 2017, respectivamente. El deterioro en la predicción para el resto de años podría deberse a la variabilidad de los datos, ocasionada por la inestabilidad económica del periodo de análisis. Lo descrito concuerda con Arellano, Gil y Martínez (2003) y Úsuga y Patiño (2008), autores que afirman que el análisis discriminante es sensible a esta variabilidad; mientras que la regresión logística, al ser una función exponencial, es más robusta y reacciona mejor.

Los resultados muestran que las microempresas son las más propensas a tener estrés financiero; hecho que corresponde con los datos de la Superintendencia de Bancos (2020), que evidencian que los créditos otorgados para microempresas son superiores a los créditos destinados a pequeñas, medianas y grandes empresas. Por lo tanto, las microempresas tienen mayor necesidad de fondos. En cierto modo, el otorgamiento de crédito (independientemente del tipo de empresa) no necesariamente es saludable, ya que la rentabilidad acumulada, la solvencia, la rentabilidad, la cobertura de la deuda y el capital de trabajo de las ECE son bajos. Al final, el otorgamiento de crédito contribuiría a redundar en una situación crítica, no por el crédito en sí, sino por las circunstancias que desencadenaría, dadas las características de la microempresas (García et al., 2018). Desde luego, lo expuesto no es más que una suposición razonable, ya que - en estricto sentido - tendría que contrastarse mediante una prueba de hipótesis. Sin embargo, no se cuenta con los datos necesarios para este fin, y el propósito de este estudio es otro. De cualquier modo, sería conveniente cubrir dicho asunto en futuras investigaciones.

Finalmente, las oscilaciones en el comportamiento del estrés financiero -en el 2015 y el 2016 en particular, y en todo el periodo en general- podrían ser causadas por los cambios de los precios de las materias primas que se dieron en el ámbito global en esos años, si no por completo, sí en parte. La crisis significó la caída del precio del petróleo y la apreciación del dólar, lo cual impactó directamente en el PIB ecuatoriano y, en consecuencia, en el sector manufacturero. Las dificultades se materializaron en el cumplimiento de obligaciones, por un lado, y en el acceso al crédito, por otro. Así, en el 2016 se registraron 7294 empresas menos que el año anterior (DIEE, 2018). Los modelos y los resultados posteriores con base en estos evidencian esta crítica situación.

\section{CONCLUSIONES}

Además del aporte académico, en el presente trabajo existe uno pragmático: potenciales directrices tanto para el sector empresarial como para los tomadores de 
decisiones de políticas públicas. Se establece un diagnóstico que contribuye en la mitigación del deterioro de indicadores y la limitación en el crédito futuro, que potencialmente podría dar paso a las ECE.

Un hallazgo importante es que las microempresas muestran mayor estrés financiero, lo cual evidencia la debilidad de estas entidades y concuerda con los planteamientos de Männasoo (2008). Los resultados invitan a los microempresarios a tomar medidas frente al endeudamiento ya que, pese a ser imprescindible para el crecimiento, constituye un riesgo potencial para la empresa. Las medidas que vayan a adoptarse deben corresponder al tipo de empresa. Es fundamental tener especial atención con las microempresas y con las provincias en las que estas se ubican.

Finalmente, llama la atención el sentido de los signos de algunos modelos estimados en este estudio. Esto es indicativo de las características en extremo diferentes del contexto ecuatoriano, sinónimo de subdesarrollo en última instancia -o de "en vías de desarrollo", si se quiere una óptica distinta del mismo punto-. Por lo tanto, la reestimación de los coeficientes es acertada; emplear aquellos obtenidos para EUA, por ejemplo, sería inapropiado, por decir menos.

\section{REFERENCIAS}

1. Abeles, M., Cimoli, M. y avarello, P. (2017). Manufactura y cambio estructural. Santiago de Chile: Comisión Económica para América Latina y el Caribe (CEPAL).

2. Agarwal, V. y Taffler, R. (2008). Comparing the performance of market-based and accounting-based bankruptcy prediction models. Journal of Banking and Finance, 32 (8), 1541-1551. https:/doi.org/10.1016/j.jbankfin.2007.07.014

3. Ahmad, A. H. (2019). What factors discriminate reorganized and delisted distressed firms: Evidence from Malaysia. Finance Research Letters, 29, 50-56. https:/doi. org/10.1016/j.frl.2019.03.010

4. Alaka, H. A., Oyedele, L. O., Owolabi, H. A., Kumar, V., Ajayi, S. O., Akinade, O. O. y Bilal, M. (2018). Systematic review of bankruptcy prediction models: Towards a framework for tool selection. Expert Systems with Applications, 94, 164-184. https:/ doi.org/10.1016/j.eswa.2017.10.040

5. Altman, E. I. (1968). Financial ratios, discriminant analysis and the prediction of corporate bankruptcy. The Journal of Finance, 23(4), 589-609. https:/doi. org/10.1111/j.1540-6261.1968.tb00843.x

6. Altman, E. I. (2000). Predicting financial distress of companies: revisiting the Z-Score and ZETA ${ }^{\circledR}$ models. Handbook of Research Methods and Applications in Empirical Finance, (September, 1968), 428-456. https:/doi.org/10.4337/9780857936097.00027 
Freddy Benjamín Naula-Sigua • Diana Jackeline Arévalo-Quishpi • Jorge Andrés Campoverde-Picón • Josselyn Patricia López-González

7. Altman, E. I. y Hotchkiss, E. (2005). Corporate financial distress and bankruptcy: Predict and avoid bankruptcy analyze and invest in distressed debt (3. - ed.). Nueva York: John Wiley \& Sons. https:/doi.org/10.1002/9781118267806.ch11

8. Altman, E. I. y Hotchkiss, E. (2006). Corporate Financial Distress and Bankruptcy (3.a ed.). New Jersey: Wiley Finance Series.

9. Altman, E. I., Danovi, A. y Falini, A. (1988). Z-Score Models' Application to Italian companies subject to extraordinary administration. Journal of Applied Finance. Formerly Finance Practice and Education, 23(1), 10.

10. Altman, E. I., Laitinen, E. K. y Suvas, A. (2017). Financial distress prediction in an international context: A review and empirical analysis of Altman's Z-Score Model. Journal of International Financial Management $\mathcal{E}$ Accounting, 28(2), 131-172. https:/ doi.org/10.1111/jifm.12053

11. Altman, I. (1994). Corporate distress diagnosis: Comparisons using linear discriminant analysis and neural networks (the Italian experience). Journal of Banking and Finance, 18(3), 505-529. https:/doi.org/10.1016/0378-4266(94)90007-8

12. Arellano, A. S., Gil, J. A. y Martínez, A. H. (2003). El análisis discriminante en la previsión de la insolvencia en las empresas de seguros de no vida. Revista Española de Financiación y Contabilidad, 32(116), 183-233 https:/doi.org/10.1080/02102412.2 003.10779487

13. Back, B., Laitinen, T., Sere, K. y van Wezel, M. (2009). Choosing bankruptcy predictors using discriminant analysis, logit analysis and genetic algorithms. Turku, Centre for Computer Science, 40, 214. https://citeseerx.ist.psu.edu/viewdoc/download?doi=10.1. 1.604.2813\&rep $=$ rep1\&type $=$ pdf

14. Bae, J. K. (2012). Predicting financial distress of the South Korean manufacturing industries. Expert Systems with Applications, 39(10), 9159-9165. https:/doi.org/10.1016/j. eswa.2012.02.058

15. Baidya, T., Ribeiro, L. y Altman, E. I. (1979). Assessing potential financial problems for firms in Brazil. Journal of International Business Studies, 10(2), 9-24. https:/doi. org/10.1057/palgrave.jibs.8490787

16. Balcaen, S. y Ooghe, H. (2006). 35 years of studies on business failure: An overview of the classic statistical methodologies and their related problems. British Accounting Review, 38(1), 63-93. https:/doi.org/10.1016/j.bar.2005.09.001

17. Banco Central del Ecuador (2019). Información Estadística Mensual 2006 [abril]. https:// contenido.bce.fin.ec/home1/estadisticas/bolmensual/IEMensual.jsp

18. Banco Mundial (2017). Políticas procíclicas vs. Políticas contracíclicas. https://www.bancomundial.org/es/news/infographic/2017/10/12/politicas-prociclicas-politicas-contraciciclas.

19. Bartoloni, E. y Baussola, M. (2014). Financial performance in manufacturing firms: A comparison between parametric and non-parametric approaches. Business Economics, 49(1), 32-45. https://ideas.repec.org/a/pal/buseco/v49y2014ilp32-45.html

20. Beaver, W. H. (1966). Financial ratios as predictors of failure. Journal of Accounting Research, 4(71). https:/doi.org/10.2307/2490171 
21. Beaver, W., McNichols, M. y Rhie, J. W. (2005). Have financial statements become less informative? Evidence from the ability of financial ratios to predict bankruptcy. Review of Accounting Studies, 10(1), 93-122. https:/doi.org/10.1007/s11142-004-6341-9

22. Bhattacharya, H. (2007). Total management by ratios (2. ${ }^{\mathrm{a}}$ ed.). Sage Publications India.

23. Blum, M. (1974). Failing company discriminant analysis. Journal of Accounting Research, 12(1), 1. https:/doi.org/10.2307/2490525

24. Brealey, R. A., Myers, S. C. y Allen, F. (2011). Principles of corporate finance (10. eㅡ.). Nueva York: McGraw Hill.

25. Campbel, J. Y., Hilscher, J. y Szilagyi, J. (2008). In search of distress risk. Journal of Finance, 63(6), 2899-2939.

26. Carmichael, D. R. (1972). The Auditor's reporting obligation: The meaning and implementation of the fourth standard of reporting. Auditing research monograph (8.ㄹ ed.). Guides, Handbooks and Manuals.

27. Chan, K. C. y Chen, N. F. (1991). Structural and return characteristics of small and large firms. The Journal of Finance, 46(4), 1467-1484.

28. Chen, Y., Zhang, L. y Zhang, L. (2013). Financial Distress Prediction for Chinese Listed Manufacturing Companies. Procedia Computer Science, 17, 678-686. https:/doi. org/10.1016/j.procs.2013.05.088

29. Chin, B. y Yap, F. (2012). Evaluating company failure in malaysia using financial ratios and logistic regression. Asian Journal of Finance $\mathcal{E}$ Accounting, 4(1), 330-344. https:/ doi.org/10.5296/ajfa.v4i1.1752

30. Comisión Económica para América Latina y el Caribe (CEPAL) (2017). CEPALSTAT. Perfil económico ALC. https://estadisticas.cepal.org/cepalstat/Perfil_Regional_ Economico.html?idioma $=$ spanish

31. Comisión Económica para América Latina y el Caribe (CEPAL) (2008). Estudio económico de América Latina y el Caribe. https://www.cepal.org/es/publicaciones/1068 estudio-economico-america-latina-caribe-2008-2009-politicas-la-generacion-empleo

32. Danenas, P. y Garsva, G. (2012). Credit Risk modeling of USA manufacturing companies using linear SVM and Sliding Window Testing Approach. Business Information Systems, 15, 249-259. https:/doi.org/10.1007/978-3-642-20511-8

33. Davig, T. y Hakkio, C. (2010). What is the effect of financial stress on economic activity. Economic Review. Federal Reserve Bank of Kansas City, 95 (Q II), 35-62. http:// ideas.repec.org/a/fip/fedker/y2010iqiip35-62nv.95no.2.html

34. Deakin, E. B. (1972). A Discriminant analysis of predictors of business failure. Journal of Accounting Research, 10(1), 167. https:/doi.org/10.2307/2490225

35. Dimitras, A. I., Zanakis, S. H. y Zopounidis, C. (1996). A survey of business failures with an emphasis on prediction methods and industrial applications. European Journal of Operational Research, 90(3), 487-513. https:/doi.org/10.1016/0377-2217(95)00070-4

36. Dirección de Estadísticas Económicas (DIEE) (2018). Directorio de Empresas y Establecimientos, 2017. Boletín técnico, 1-2018. Quito: DIEE. 
Freddy Benjamín Naula-Sigua • Diana Jackeline Arévalo-Quishpi • Jorge Andrés Campoverde-Picón • Josselyn Patricia López-González

37. Dirección Nacional de Investigación y Estudios (DNIYE) (Coord.) (2018). Panorama de la industria manufacturera en Ecuador, 2013-2017. https://investigacionyestudios.supercias.gob.ec/wp-content/uploads/2018/09/Panorama-de-la-Industria-Manufactureraen-el-Ecuador-2013-2017.pdf

38. Dudley, E. y Ellie, Q. (2018). Financial distress, refinancing, and debt structure. Journal of Banking and Finance, 94, 185-207. https:/doi.org/10.1016/j.jbankfin.2018.07.004

39. Edmister, R. O. (1972). an empirical test of financial ratio analysis for small business failure prediction. The Joumal of Financial and Quantitative Analysis, 7(2), 1477. https:/ doi.org/10.2307/2329929

40. Ehrhardt, M. C. y Brigham, E. F. (2007). Finanzas corporativas (2.ㄹ ed.). Ciudad de México: CENGAGE Learning.

41. Eom, Y. H., Kim, D. W. y Altman, E. I. (1998). Failure prediction: Evidence from Korea. Journal of International Financial Management and Accounting, 6.

42. Espinel, K. (2016). Riesgo de quiebra empresarial en el Ecuador durante 2009 a 2012. Universidad de Las Américas.

43. Etheridge, H. L. y Sriram, R. S. (1997). A comparison of the relative costs of financial distress models: artificial neural networks, logit and multivariate discriminant analysis. International Journal of Intelligent Systems in Accounting, Finance E Management, 6(3), 235 248. https:/doi.org/10.1002/(sici) 1099-1174(199709)6:3 < 235::aid-isaf135>3.0.co;2-n

44. Fama, E. F. y French, K. R. (1992). The Cross-Section of Expected Stock Returns. The Journal of Finance, 47(2), 427-465. https:/doi.org/10.1017/CBO9781107415324.004

45. Fama, E. F. y French, K. R. (1993). Common risk factors in the returns on stocks and bonds. Journal of Financial Economics, 33(1), 3-56. https:/doi.org/10.1016/0304 $-405 X(93) 90023-5$

46. Fernández-Gámez, M. Á., Soria, J. A. C., Santos, J. A. y Alaminos, D. (2020). European country heterogeneity in financial distress prediction: An empirical analysis with macroeconomic and regulatory factors. Economic Modelling, 88(September), 398-407. https:/doi.org/10.1016/j.econmod.2019.09.050

47. Foulke, R. A. (1968). Practical financial statement analysis. Nueva York: McGraw-Hill Company.

48. Frydman, H., Altman, E. I. y Kao, D. L. (1985). Introducing recursive partitioning for financial classification: The case of financial distress. The Journal of Finance, 40(1), 269-291.

49. García, M., Ollague, J. y Capa, L. (2018). La realidad crediticia para las pequeñas y medianas empresas ecuatorianas. http://scielo.sld.cu/scielo.php?script =sci_arttext\&pid $=\mathrm{S} 2218-36202018000200040 \# \mathrm{~B} 4$

50. Gitman, L. (1981). Fundamentos de administración financiera. Ciudad de México: Harper \& Row Latinoamerican.

51. Gordon, M. J. (1964). Postulates, principles and research in accounting. The Accounting Review, 39(2), 251-263. 
52. Gregova, E., Valaskova, K., Adamko, P., Tumpach, M. y Jaros, J. (2020). Predicting financial distress of slovak enterprises: Comparison of selected traditional and learning algorithms methods. Sustainability, 12 (10), 1-17. https:/doi.org/10.3390/SU12103954

53. Guresen, E. y Kayakutlu, G. (2011). Definition of artificial neural networks with comparison to other networks. Procedia Computer Science, 3, 426-433. https:/doi. org/10.1016/j.procs.2010.12.071

54. Hair, J. F., Black, W. C., Babin, B. J. y Anderson, R. E. (2014). Multivariate data analysis (7. ${ }^{\mathrm{a}}$ ed.). Nueva York: Pearson.

55. Herbert, H. (1985). Economic Aspects Bankruptcy Law. Journal of Institutional and Theoretical Economics, 141, 80-98.

56. Hernández Ramírez, M. (2014). A financing guideline for the detection of bankruptcy with the aid of a multiple discriminating analysis. Intersedes: Revista de las Sedes Regionales, 15(32), 4-19. http://www.redalyc.org/pdf/666/66633023001.pdf

57. Horváthová, J. y Mokrišová, M. (2020). Comparison of the results of a data envelopment analysis model and logit model in assessing business financial health. Information, 11 (3), 1-20. https:/doi.org/10.3390/info11030160

58. Hua, Z., Wang, Y., Xu, X., Zhang, B. y Liang, L. (2007). Predicting corporate financial distress based on integration of support vector machine and logistic regression. Expert Systems with Applications, 33(2), 434-440. https:/doi.org/10.1016/j.eswa.2006.05.006

59. Hyz, A. (2019). SME finance and the economic crisis: The case of Greece. Routledge

60. Ibarra, A. (2001). Análisis de las dificultades financieras de las empresas en una economía emergente: Las bases de datos y las variables independientes en el sector hotelero de la bolsa mexicana de valores. Barcelona: Universitat Autonoma de Barcelona. http://www. eumed.net/tesis/2010/aim/index.htm

61. Isik, O., Jones, M. y Sidorova, A. (2012). Using neural nets to combine information sets in corporate bankruptcy prediction. Intelligent Systems in Accounting, Finance and Management, 19, 90-101. https:/doi.org/10.1002/isaf

62. Ko, Y. C., Fujita, H. y Li, T. (2017). An evidential analysis of Altman Z-score for financial predictions: Case study on solar energy companies. Applied Soft Computing Journal, 52, 748-759. https:/doi.org/10.1016/j.asoc.2016.09.050

63. Koller, T., Goedhart, M. y Wesseles, D. (2020). Valuation, measuring and managing the value of companies. (McKinsey y Company, ed.) (7. ㄹ ed.). Hoboken: John Wiley \& Sons.

64. Kolodner, J. (1993). Case-based reasoning. San Mateo: Morgan Kaufmann Publisher.

65. Laitinen, E. K. y Laitinen, T. (1998). Cash management behavior and failure prediction. Journal of Business Finance and Accounting, 25(7-8), 893-919. https:/doi. org/10.1111/1468-5957.00218

66. Lev, B. (1969). Industry averages as targets for financial ratios. Journal of Accounting Research, 7(2), 290-299.

67. Liang, D., Lu, C., Tsai, C. y Shih, G. (2016). Financial ratios and corporate governance indicators in bankruptcy prediction: A comprehensive study. European Journal of Operational Research, 252(2), 561-572. https:/doi.org/10.1016/j.ejor.2016.01.012 
Freddy Benjamín Naula-Sigua • Diana Jackeline Arévalo-Quishpi • Jorge Andrés Campoverde-Picón • Josselyn Patricia López-González

68. Lin, T. H. (2009). A cross model study of corporate financial distress prediction in Taiwan: Multiple discriminant analysis, logit, probit and neural networks models. Neurocomputing, 72(16-18), 3507-3516. https:/doi.org/10.1016/j.neucom.2009.02.018

69. Männasoo, K. (2008). Patterns of firm survival in Estonia. Eastern European Economics, 46(4), 27-42. https:/doi.org/10.2753/EEE0012-8775460402

70. Margaine, M., Schlosser, M., Vernimmen, P. y Altman, E. I. (1974). Financial and statistical analysis for commercial loan evaluation: A French experience. Journal of Financial and Quantitative Analysis, 9(02), 195-211.

71. Maricica, M. y Georgeta, V. (2012). Business failure risk analysis using financial ratios. Procedia. Social and Behavioral Sciences, 62, 728-732. https:/doi.org/10.1016/j. sbspro.2012.09.123

72. Molina, C. (2017). ¿Por qué se endeudan las empresas latinoamericanas? Debates IESA, (2014), 46-49.

73. Mora, A. (1994). Limitaciones metodológicas de los trabajos empíricos sobre la predicción del fracaso empresarial. Revista Española de Financiación y Contabilidad, 24(80), 709-732.

74. Mselmi, N., Lahiani, A. y Hamza, T. (2017). Financial distress prediction: The case of French small and medium-sized firms. International Review of Financial Analysis, 50, 67-80. https:/doi.org/10.1016/j.irfa.2017.02.004

75. Müller, A. C. y Guido, S. (2016). Introduction to Machine Learning with Python. A guide for data scientists. Sebastopol: O'Reilly Media.

76. Nandi, A., Sengupta, P. P. y Dutta, A. (2019). Diagnosing the financial distress in oil drilling and exploration sector of india through discriminant analysis. Vision, 1-10. https:/doi.org/10.1177/0972262919862920

77. Nyitrai, T. y Virág, M. (2019). The effects of handling outliers on the performance of bankruptcy prediction models. Socio-Economic Planning Sciences, 67, 34-42. https:/doi. org/10.1016/j.seps.2018.08.004

78. Ohlson, J. A. (1980). Financial ratios and the probabilistic prediction of bankruptcy. Journal of Accounting Research, 18(1), 109. https:/doi.org/10.2307/2490395

79. Orellana, I., Reyes, M. y Cevallos, E. (2018). Análisis de insolvencia del sector alimenticio de la ciudad de Cuenca. Observatorio empresarial. Universidad del Azuay, 73-92. https:/doi.org/10.1017/CBO9781107415324.004

80. Oude Avenhuis, J. (2013). Testing the generalizability of the bankruptcy prediction models of Altman, Ohlson and Zmijewski for Dutch listed and large non-listed firms. Universidad of Twente. The School of Management and Governance.

81. Palepu, K. G., Healy, P. M. y Bernard, V. L. (2003). Business Analysis Eु Valuation: Using Financial Statements (3. - ed.). Nueva York: South-Western College Pub.

82. Park, C. S. y Han, I. (2002). A case-based reasoning with the feature weights derived by analytic hierarchy process for bankruptcy prediction. Expert Systems with Applications, 23(3), 255-264. https:/doi.org/10.1016/S0957-4174(02)00045-3 
83. Pham Vo Ninh, B., Do Thanh, T. y Vo Hong, D. (2018). Financial distress and bankruptcy prediction: An appropriate model for listed firms in Vietnam. Economic Systems, 42(4), 616-624. https:/doi.org/10.1016/j.ecosys.2018.05.002

84. Pillajo, V., Salas, S. y Palacios, J. (2018). Modelo Z de Altman: predictor de quiebras.

85. Pindado, J., Rodrigues, L. y De la Torre, C. (2016). How does financial distress affect small firm's financial structure? Small Business Economics, 26(4), 377-391.

86. Pongsatat, S., Ramage, J. y Lawrence, H. (2004). Bankruptcy Prediction for Large and Small Firms in Asia : A Comparison of Ohlson and Altman. Journal of Accounting and Corporate Governance, 1, 1-13.

87. Pozzoli, M. y Paolone, F. (2017). Corporate Financial Distress: A Study of the Italian Manufacturing Industry. Cham, Switzerland: Springer. http://search.ebscohost.com/login.aspx?direct $=$ true $\& d b=$ edsebk \&AN $=1594211 \& a m p \% 0 A$ lang $=$ pt-pt\&site $=$ eds-live $\&$ authtype $=$ sso

88. Ramayah, T., Ahmad, N. H., Halim, H. A., Rohaida, S., Zainal, M. y Lo, M. (2010). Discriminant analysis: An illustrated example. African Journal of Business Management, 4(9), 1654-1667.

89. Rifqi, M. y Kanazaki, Y. (2016). Predicting financial distress in indonesian manufacturing industry. Sendai: Tohoku Management \& Accounting Research Group.

90. Ross, S. A., Westerfield, R. y Jordan, B. D. (2017). Essentials of corporate finance (9.a ed.). Nueva York: McGraw-Hill Education.

91. Ross, S., Westerfield, R. y Jordan, B. (2010). Fundamentos de finanzas corporativas (9. a ed.). México: McGraw Hill.

92. Sayari, N. y Mugan, C. S. (2017). Industry specific financial distress modeling. BRQ Business Research Quarterly, 20(1), 45-62. https:/doi.org/10.1016/j.brq.2016.03.003

93. Serrano, C., Gutiérrez, B. y Bernate, M. (2019). The use of accounting anomalies indicators to predict business failure. European Management Journal, 37(3), 353-375. https:/doi.org/10.1016/j.emj.2018.10.006

94. Shilpa, N. C. y Amulya, M. (2017). Corporate financial distress: Analysis of Indian automobile industry. SDMIMD Journal of Management, 8(1), 85. https:/doi.org/10.18311/ sdmimd/2017/15726

95. Subramanyam, K. R. y Wild, J. J. (2009). Financial Statement Analysis (10.ㄹ ed.). Nueva York: McGraw-Hill/Irwin.

96. Sun, J., Li, H., Huang, Q. H. y He, K. Y. (2014). Predicting financial distress and corporate Failure: A review from the state-of-the-art definitions, modeling, sampling, and featuring approaches. Knowledge-Based Systems, 57, 41-56. https:/doi.org/10.1016/j. knosys.2013.12.006

97. Superintendencia de Bancos. (2020). Estudios y Análisis. Portal estadístico. https:// estadisticas.superbancos.gob.ec/portalestadistico/portalestudios/?page_id=1054\# 1508173151736-7ddba919-ba8c

98. Swanson, E. V. y Tybout, J. R. (1988). Industrial bankruptcy determinants in Argentina. International Business Failure Prediction Models, 7. 
Carlos David Cardona-Arenas • Lya Paola Sierra-Suárez • Jorge Andrés Campoverde-Picón • Josselyn Patricia López-González

99. Takahashi, K., Kurokawa, Y. y Watase, K. (1984). Corporate bankruptcy prediction in Japan. Journal of Banking and Finance, 8(2), 229-247. https:/doi. org/10.1016/0378-4266(84)90005-0

100. Trueck, S. y Rachev, S. (2009). Rating and scoring techniques. Rating Based Modeling of Credit Risk. https:/doi.org/10.1016/b978-0-12-373683-3.00003-8

101. Úsuga Manco, O. C. y Patiño Rodríguez, C. E. (2008). Análisis discriminante no métrico y regresión logística en el problema de clasificación. TecnoLógicas, (21), 13-29. https:/doi.org/10.22430/22565337.249

102. Valencia Cárdenas, M., Trochez González, J., Vanegas López, J. G. y Restrepo Morales, J. A. (2016). Modelo para el análisis de la quiebra financiera en pymes agroindustriales antioqueñas. Apuntes del Cenes, 35 (62), 147-168. https:/doi.org/10.19053/22565779.4310

103. Veganzones, D. y Severin, E. (2020). Corporate failure prediction models in the twenty-first century: a review. European Business Review. https:/doi.org/10.1108/ EBR-12-2018-0209

104. Wang, H., Jiang, Y. y Wang, H. (2009). Stock return prediction based on Baggingdecision tree. 2009 IEEE International Conference on Grey Systems and Intelligent Services, (GSIS 2009), 1575-1580. https:/doi.org/10.1109/GSIS.2009.5408165

105. Wang, Y. y Campbell, M. (2010). Financial ratios and the prediction of bankruptcy: The Ohlson Model applied to Chinese Publicly Traded Companies. Proceedings of ASBBS, 17 (January).

106. Yazdipour, R. (2011). Advances in entrepreneurial finance. Nueva York: Springer. https:/ doi.org/10.1007/978-1-4419-7527-0

107. Zmijewski, M. E. (1984). Methodological issues related to the estimation of financial distress prediction models. Journal of Accounting Research, 22 (1984), 59-82. 